\title{
Atom-dimer and dimer-dimer scatterings in a spin-orbit-coupled Fermi gas
}

\author{
M. Iskin \\ Department of Physics, Koç University, Rumelifeneri Yolu, 34450 Sartyer, Istanbul, Turkey
}

(Received 21 October 2020; revised 12 February 2021; accepted 12 February 2021; published 26 February 2021)

\begin{abstract}
Using the diagrammatic approach, here we study how spin-orbit coupling (SOC) affects the fermion-dimer and dimer-dimer scattering lengths in the Born approximation, and we benchmark their accuracy with the higherorder approximations. We consider both isotropic and Rashba couplings in three dimensions and show that the Born approximation gives accurate results in the $1 /\left(m \alpha a_{s}\right) \ll-1$ limit, where $m$ is the mass of the fermions, $\alpha$ is the strength of the SOC, and $a_{s}$ is the $s$-wave scattering length between fermions. This is because the higher-loop contributions form a perturbative series in the $1 /\left(m \alpha a_{s}\right)<0$ region that is controlled by the smallness of the residue $Z$ of the dimer propagator. In sharp contrast, since $Z$ grows with the square root of the binding energy of the dimer in the $1 /\left(m \alpha a_{s}\right)>0$ region, all of the higher-loop contributions are of similar order.
\end{abstract}

DOI: 10.1103/PhysRevA.103.023337

\section{INTRODUCTION}

The diagrammatic approach has proven to be a powerful technique for studying few-body problems in many branches of theoretical physics. For instance, in the context of shortrange two-body interactions between particles, it has been successfully applied to both the three-body [1-7] and the four-body $[2,3,8,9]$ problems to verify the known exact results for the fermion-dimer [10,11] and dimer-dimer [12] scattering lengths, respectively. In addition, the approach has recently been generalized to the three-body problem with arbitrary-range two-body interactions and applied to the electron-exciton scattering in semiconductors, i.e., to the socalled three-body Coulomb problem [13].

Furthermore, in the context of BCS-BEC (Bose-Einsten condensate) crossover [14], the fermion-dimer and dimerdimer scattering lengths appear in some of the many-body properties of dilute Fermi gases, including their low-energy collective modes, superfluid density, etc. Such appearances are quite natural in those parameter regimes where a strongly interacting Fermi-Fermi mixture can be mapped to a weakly interacting Bose-Fermi mixture of paired (i.e., bosonic dimers) and unpaired (i.e., excess) fermions $[4,15,16]$. However, it is also known that the usual treatment of the BCS-BEC crossover through a Gaussian fluctuation approach yields fermion-dimer and dimer-dimer scattering lengths that are consistent with the lowest-order Born approximation $[2,3,8]$.

Given the recent surge of experimental [17-20] and theoretical [21-27] interests in spin-orbit-coupled Fermi gases, here we extend the diagrammatic approach to the relevant few-body problems. In particular, we study how spin-orbit coupling (SOC) affects the fermion-dimer and dimer-dimer scattering lengths in the Born approximation, and we benchmark their accuracy with the higher-order approximations. Our primary findings for the isotropic and Rashba couplings in three dimensions are as follows. We show that the Born approximation gives accurate results in the $1 /\left(m \alpha a_{s}\right) \ll-1$ limit, as the higher-loop contributions form a perturbative series in the $1 /\left(m \alpha a_{s}\right)<0$ region that is controlled by the residue $Z$ of the dimer propagator. While $Z$ decays to 0 in the $1 /\left(m \alpha a_{s}\right) \rightarrow-\infty$ limit, it grows with the square root of the binding energy of the dimer in the $1 /\left(m \alpha a_{s}\right)>0$ region, suggesting that it may be sufficient to consider a finite number of higher-loop diagrams in the $1 /\left(m \alpha a_{s}\right)<0$ region.

The rest of the paper is organized as follows. In Sec. II, we introduce the one-body Hamiltonian, helicity bands, and the fermion propagator. In Sec. III, we introduce the two-body Hamiltonian, identify the appropriate Feynman rules for the bound-state problem, and derive the dimer propagator for the composite bosons. In Sec. IV, we analyze the fermion-dimer scattering $t$ matrix and extract the fermion-dimer scattering length in the zero-loop Born, one-loop, and two-loop approximations. In Sec. V, we analyze the dimer-dimer scattering $t$ matrix and extract the dimer-dimer scattering length in the one-loop Born and two-loop approximations. In Sec. VI, we discuss how the fermion-dimer and dimer-dimer scattering lengths are related to the many-body problem. In Sec. VII, we compare our findings for the isotropic SOC with those of the anisotropic (Rashba) SOC. The paper ends with a brief summary of our conclusions in Sec. VIII. For the sake of completeness, the binding energy and effective mass of the dimer are presented in the Appendix.

\section{ONE-BODY PROBLEM}

In the $\langle\uparrow|=\left(\begin{array}{ll}1 & 0\end{array}\right)$ and $\langle\downarrow|=\left(\begin{array}{ll}0 & 1\end{array}\right)$ basis of the $\sigma_{z}$ Pauli matrix, the single-particle problem is governed by the Hamiltonian matrix

$$
h_{\mathbf{k}}=\varepsilon_{\mathbf{k}} \sigma_{0}+\alpha \mathbf{k} \cdot \boldsymbol{\sigma}
$$

in momentum space, where $\mathbf{k}=\left(k_{x}, k_{y}, k_{z}\right)$ is the wave vector, $\varepsilon_{\mathbf{k}}=k^{2} /(2 m)$ is the usual dispersion with $k=\sqrt{k_{x}^{2}+k_{y}^{2}+k_{z}^{2}}$ in units of $\hbar=1, \sigma_{0}$ is a unit matrix, $\alpha \geqslant 0$ is the strength of the SOC that is taken as an isotropic field in $\mathbf{k}$ space, and 


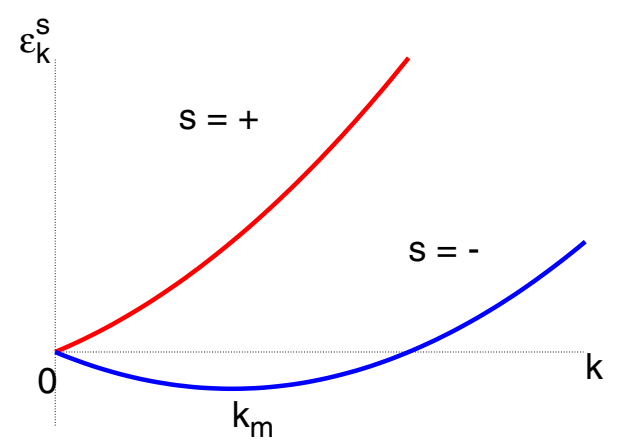

FIG. 1. One-body dispersions $\varepsilon_{\mathbf{k}}^{s}=k^{2} /(2 m)+s \alpha k$ for the $s=$ \pm helicity bands. The minimum of the lower band corresponds to a shell of $\mathbf{k}$ states with the radius $k_{m}=m \alpha$ and the energy $\varepsilon_{\mathbf{k}_{m}}^{-}=$ $-m \alpha^{2} / 2$.

$\boldsymbol{\sigma}=\left(\sigma_{x}, \sigma_{y}, \sigma_{z}\right)$ is a vector of Pauli matrices. The eigenvalues and eigenvectors of $h_{\mathbf{k}}$ are determined by the unitary transformation

$$
U_{\mathbf{k}}=\frac{1}{\sqrt{2 k\left(k-k_{z}\right)}}\left(\begin{array}{cc}
k_{x}-i k_{y} & k_{z}-k \\
k-k_{z} & k_{x}+i k_{y}
\end{array}\right),
$$

where $U_{\mathbf{k}}^{\dagger} h_{\mathbf{k}} U_{\mathbf{k}}$ gives the dispersion relations of the $s= \pm$ helicity bands

$$
\varepsilon_{\mathbf{k}}^{s}=\frac{k^{2}}{2 m}+s \alpha k,
$$

and $U_{\mathbf{k}}|\uparrow(\downarrow)\rangle$ gives the corresponding eigenstates. We illustrate these dispersions in Fig. 1 as a function of $k$, and note that the ground state of the --helicity band corresponds to a degenerate shell of $\mathbf{k}$ states with the radius $k_{m}=m \alpha$ and energy $\varepsilon_{\mathbf{k}_{m}}^{-}=-m \alpha^{2} / 2$.

Given the Hamiltonian matrix in Eq. (1), the propagator of the single particle can be written as

$$
G\left(\mathbf{k}, k_{0}\right)=\frac{1}{\left(k_{0}+i 0^{+}\right) \sigma_{0}-h_{\mathbf{k}}},
$$

where $k_{0}$ is the energy, and we set the chemical potential $\mu$ to 0 for the few-body problems of interest below. In our analysis, we reexpress such propagators via the generic relation $1 /\left(A \sigma_{0}-\mathbf{B} \cdot \boldsymbol{\sigma}\right)=\left(A \sigma_{0}+\mathbf{B} \cdot \boldsymbol{\sigma}\right) /\left(A^{2}-\right.$ $\left.B^{2}\right)=(1 / 2) \sum_{s}\left(\sigma_{0}+s \widehat{\mathbf{B}} \cdot \boldsymbol{\sigma}\right) /(A-s B)$, where $\widehat{\mathbf{B}}=\mathbf{B} / B$ and $B=|\mathbf{B}|$.

\section{TWO-BODY PROBLEM}

Having in mind the atomic Fermi gases where the bosonic dimer is a result of a short-range interaction between $\uparrow$ and $\downarrow$ fermions, our two-body interaction is governed by the Hamiltonian density

$$
h_{\mathbf{r}}=-g \psi_{\uparrow}^{\dagger}(\mathbf{r}) \psi_{\downarrow}^{\dagger}(\mathbf{r}) \psi_{\downarrow}(\mathbf{r}) \psi_{\uparrow}(\mathbf{r})
$$

in real space, where $g \geqslant 0$ is the strength of the fermionfermion attraction, and $\psi_{\sigma}^{\dagger}(\mathbf{r})$ and $\psi_{\sigma}(\mathbf{r})$ are the fermionic field operators. A convenient way to understand the action of this term is through a Hubbard-Stratonovich transformation in the imaginary-time functional path-integral formalism [22-27]. Introducing the Hubbard-Stratonovich fields $\Delta=$ $-g \psi_{\downarrow} \psi_{\uparrow}$ and $\bar{\Delta}=-g \bar{\psi}_{\uparrow} \bar{\psi}_{\downarrow}$, where $\bar{\psi}_{\sigma}$ and $\psi_{\sigma}$ are the

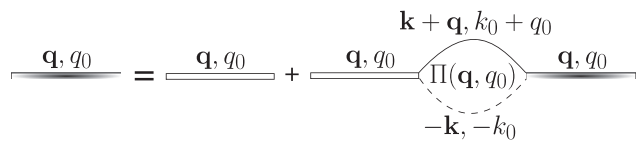

FIG. 2. Diagrammatic representation of the two-body binding problem. The dimer propagator (colored bars) is determined by dressing its bare propagator (uncolored bars) with infinitely many fermion-fermion bubbles (solid and dashed lines), forming eventually a geometric series.

corresponding Grassmann variables with suppressed arguments $x=(\mathbf{r}, \tau)$ for notational simplicity, the action that corresponds to Eq. (5) is replaced by three terms: $\bar{\Delta} \Delta / g+$ $\Delta \bar{\psi}_{\uparrow} \bar{\psi}_{\downarrow}+\bar{\Delta} \psi_{\downarrow} \psi_{\uparrow}$. If one interprets $\bar{\Delta}=\Delta^{*}$ as the complex dimer field, then the first term describes free dimers with a bare propagator $-g$, and the second and third terms describe the dimer-fermion and fermion-dimer conversion processes, respectively.

The diagrammatic representation of the two-body binding problem in $\mathbf{k}$ space is shown in Fig. 2, where the physical dimer propagator $D\left(\mathbf{q}, q_{0}\right)$ is determined by dressing its bare value, which is a constant in space, with repeated interactions between its fermionic constituents [2,3]. The resultant geometric series can be summed over to yield

$$
D\left(\mathbf{q}, q_{0}\right)=\frac{-g}{1+g \Pi\left(\mathbf{q}, q_{0}\right)},
$$

where $\Pi\left(\mathbf{q}, q_{0}\right)$ corresponds to the fermion-fermion bubble diagram that is given by

$$
\Pi\left(\mathbf{q}, q_{0}\right)=\frac{\operatorname{Tr}}{2} \sum_{k} \sigma_{y} G\left(\mathbf{k}+\mathbf{q}, k_{0}+q_{0}\right) \sigma_{y} G^{\mathrm{T}}\left(-\mathbf{k},-k_{0}\right) .
$$

Here, $\operatorname{Tr}$ is a trace over the spin sector, and $\sum_{k}$ represents $\sum_{\mathbf{k}, k_{0}}=i \int d^{3} \mathbf{k} \int d k_{0} /(2 \pi)^{4}$. In our diagrams, while the solid lines correspond to the fermion propagators that are described by Eq. (4), the dashed lines correspond to their dimer partners that are described by the transpose T of Eq. (4). This is because the dimer is formed between a particle that is governed by $h_{\mathbf{k}+\mathbf{q}}$ and a hole that is governed by $-h_{-\mathbf{k}}^{\mathrm{T}}$ in $\mathbf{k}$ space [22-27]. In accordance with the Feynman rules, each fermion line, dimer line, and vertex carries a factor of $i$. In addition, we associate each dimer-creation (-annihilation) vertex with an additional factor of $\mp i \sigma_{y}$ to account for the fermion-dimer (dimer-fermion) conversion terms, i.e., $-i \bar{\Delta} \sigma_{y}$ and $i \Delta \sigma_{y}$, respectively, in the particle-hole sectors.

Noting the relation $\sigma_{y} \mathbf{B} \cdot \boldsymbol{\sigma}^{\mathrm{T}} \sigma_{y}=-\mathbf{B} \cdot \boldsymbol{\sigma}$, and integrating $k_{0}$ in the upper half plane in which there are two simple poles at $k_{0}=-\varepsilon_{\mathbf{k}}^{ \pm}$, we find [25]

$$
\Pi\left(\mathbf{q}, q_{0}\right)=\frac{1}{4} \sum_{s s^{\prime} \mathbf{k}} \frac{1+s s^{\prime} \widehat{\mathbf{k}} \cdot \widehat{\mathbf{Q}}}{q_{0}-\varepsilon_{\mathbf{k}}^{s}-\varepsilon_{\mathbf{Q}}^{s^{\prime}}},
$$

where $\mathbf{Q}=\mathbf{k}+\mathbf{q}$. Equation (8) shows that only the intraband processes contribute to the bubble diagram when the dimer is stationary, i.e., when its center-of-mass momentum q vanishes. Therefore, we can reexpress the stationary bubble diagram as $\Pi\left(\mathbf{0}, q_{0}\right)=(1 / 2) \sum_{s k} G_{s}\left(\mathbf{k}, k_{0}+q_{0}\right) G_{s}\left(-\mathbf{k},-k_{0}\right)$, 
where

$$
G_{s}\left(\mathbf{k}, k_{0}\right)=\frac{1}{k_{0}-\varepsilon_{\mathbf{k}}^{s}+i 0^{+}}
$$

is the fermion propagator in the $s= \pm$ helicity basis.

In the lowest order in $\mathbf{q}$ and $q_{0}$, Eq. (6) has the generic structure of a simple pole [22-27]

$$
D\left(\mathbf{q}, q_{0}\right)=\frac{Z}{q_{0}-q^{2} /\left(2 m_{B}\right)+\mu_{B}+i 0^{+}},
$$

where $Z=8 \pi\left(\left|\varepsilon_{b}\right|-m \alpha^{2}\right)^{3 / 2} /\left(m \sqrt{m}\left|\varepsilon_{b}\right|\right)$ corresponds to the residue of the pole, $2 m / m_{B}=7 / 3-4\left(1-m \alpha^{2} /\left|\varepsilon_{b}\right|\right)^{3 / 2} / 3-$ $2 m \alpha^{2} /\left|\varepsilon_{b}\right|$ determines the effective mass of the bosonic dimer, and $\mu_{B}=2 \mu-\varepsilon_{b} \rightarrow-\varepsilon_{b}$ corresponds to its chemical potential. Noting that $-m \alpha^{2}$ is the two-body continuum threshold, the energy of the two-body bound state $\varepsilon_{b} \leqslant-m \alpha^{2}$ or the two-body binding energy $\left|\varepsilon_{b}\right|-m \alpha^{2}$ of the dimer can be simply found by looking at the pole of $D\left(\mathbf{0}, \varepsilon_{b}\right)$, leading to the relation $1=(g / 2) \sum_{s \mathbf{k}} 1 /\left(2 \varepsilon_{\mathbf{k}}^{s}-\varepsilon_{b}\right)$. In addition, we substitute $g$ with the usual $t$-matrix relation between two fermions in vacuum without the SOC, $1 / g=$ $-m V /\left(4 \pi a_{s}\right)+\sum_{\mathbf{k}} 1 /\left(2 \varepsilon_{\mathbf{k}}\right)$, where $\sum_{\mathbf{k}}=V \int d^{3} \mathbf{k} /(2 \pi)^{3}$ in units of $V=1$. This leads to $\varepsilon_{b}=-2 m \alpha^{2}-1 /\left(2 m a_{s}^{2}\right) \pm$ $\sqrt{1 /\left(4 m^{2} a_{s}^{4}\right)+\alpha^{2} / a_{s}^{2}}$ for $a_{s} \lessgtr 0$, showing that $\varepsilon_{b} \leqslant-m \alpha^{2}$ for all parameters. This expression is analytically tractable in three limits [22,25-27]: we find that (i) $\varepsilon_{b}=-m \alpha^{2}-m^{3} \alpha^{4} a_{s}^{2}$ and $m_{B}=6 m$ in the limit when $1 /\left(m \alpha a_{s}\right) \ll-1$, (ii) $\varepsilon_{b}=$ $-2 m \alpha^{2}$ and $m_{B}=3 \sqrt{2} /(2 \sqrt{2}-1) m \approx 2.32 m$ in the unitarity limit when $1 /\left(m \alpha a_{s}\right)=0$, and (iii) $\varepsilon_{b}=-1 /\left(m a_{s}^{2}\right)$ and $m_{B}=2 m$ in the limit when $1 /\left(m \alpha a_{s}\right) \gg 1$. These results are illustrated in Fig. 10 for the completeness of the presentation. Note that the latter limit recovers the usual two-body problem with no SOC in the $1 /\left(m \alpha a_{s}\right) \gg 1$ limit when $\alpha \rightarrow 0^{+}$.

\section{THREE-BODY PROBLEM}

In this section, we are interested in the scattering $t$ matrix $t_{k}^{--}(0)$ between the lowest-energy fermion in the --helicity band and a stationary dimer. For this purpose, we introduce a shorthand notation,

$$
\mathcal{T}_{k}(p)=\left[\begin{array}{cc}
t_{k}^{\uparrow \uparrow}(p) & t_{k}^{\uparrow \downarrow}(p) \\
t_{k}^{\downarrow \uparrow}(p) & t_{k}^{\downarrow \downarrow}(p)
\end{array}\right],
$$

where $k=\left(\mathbf{k}, k_{0}\right)$ refers collectively to the momentum and energy of the incoming fermion, and $p=\left(\mathbf{p}, p_{0}\right)$ refers collectively to the momentum and energy exchange between the outgoing fermion and the dimer. We refer to Fig. 5 for the clarity of its meaning. Once $\mathcal{T}_{k}(0)$ is evaluated, we transform it to the helicity basis via Eq. (2), and obtain $U_{\mathbf{k}}^{\dagger} \mathcal{T}_{k}(0) U_{\mathbf{k}}$. Using the spherical coordinates where $\widehat{\mathbf{k}}=$ $\left(\sin \theta_{\mathbf{k}} \cos \phi_{\mathbf{k}}, \sin \theta_{\mathbf{k}} \sin \phi_{\mathbf{k}}, \cos \theta_{\mathbf{k}}\right)$, we find

$$
\begin{aligned}
t_{k}^{s s}(0)= & t_{k}^{\uparrow \uparrow}(0) \frac{1+s \cos \theta_{\mathbf{k}}}{2}+t_{k}^{\downarrow \downarrow}(0) \frac{1-s \cos \theta_{\mathbf{k}}}{2} \\
& +s \operatorname{Re}\left[t_{k}^{\uparrow \downarrow}(0) \sin \theta_{\mathbf{k}}\left(\cos \phi_{\mathbf{k}}+i \sin \phi_{\mathbf{k}}\right)\right]
\end{aligned}
$$

for the diagonal elements with Re being the real part. Note in particular that $t_{k}^{--}(0)=t_{k}^{\downarrow \downarrow}(0)$ for $\mathbf{k}$ that is aligned with the $z$ axis, i.e., when $\theta_{\mathbf{k}}=0$. In this paper, we are interested in the fermion-dimer scattering length $a_{B F}$ that is determined (a)

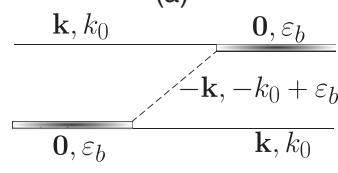

(b)

$$
\frac{\mathbf{q}-\mathbf{k}, q_{0}-\frac{\mathbf{q}, q_{0}+\varepsilon_{b}}{k_{0}+\varepsilon_{b} \quad \mathbf{q}-\mathbf{k}_{,} q_{0}-k_{0}+\varepsilon_{b}}}{\mathbf{k}-\mathbf{q}, k_{0}-q_{0}}
$$

$\frac{\mathbf{q}, q_{0}+\varepsilon_{b} \quad \mathbf{k}-\mathbf{Q}, k_{0}-Q_{0}}{\mathbf{Q}-\mathbf{k}, Q_{0}-k_{0}+\varepsilon_{b}}$

FIG. 3. Diagrammatic representations of the (a) zero-loop Born, (b) one-loop, and (c) two-loop contributions to the fermion-dimer scattering $t$ matrix.

by $[2,3]$

$$
a_{B F}=\frac{m_{B F}}{4 \pi} Z t_{k}^{--}(0),
$$

where $m_{B F}=2 m_{B} m_{F} /\left(m_{B}+m_{F}\right)$ is twice the reduced mass of the fermion and the dimer, and $k=\left(m \alpha \widehat{\mathbf{k}},-m \alpha^{2} / 2\right)$ corresponds to the lowest-energy eigenstate in the --helicity band.

For instance, the diagrammatic representations of the zeroloop, one-loop, and two-loop contributions to the fermiondimer scattering $t$ matrix are shown in Fig. $3[1-7,13]$. The zero-loop contribution is known as the Born approximation, and in accordance with the Feynman rules given above, it is given by

$$
\mathcal{T}_{k}^{(0)}(0)=-\sigma_{y} G^{\mathrm{T}}\left(-\mathbf{k},-k_{0}+\varepsilon_{b}\right) \sigma_{y},
$$

where the minus sign is due to the exchange of an identical fermion. By plugging Eq. (14) into Eq. (12), we find

$$
t_{k}^{s s(0)}(0)=\frac{1}{k_{0}-\varepsilon_{b}+\varepsilon_{\mathbf{k}}^{s}},
$$

which is physically intuitive. This is because, since both dimers are stationary in the Born diagram, the helicity bands are not coupled, and $t_{k}^{s s}(0)$ can be directly expressed as $t_{k}^{s s(0)}(0)=-G_{s}\left(-\mathbf{k},-k_{0}+\varepsilon_{b}\right)$. Furthermore, by plugging $t_{k}^{--(0)}(0)=1 /\left(\left|\varepsilon_{b}\right|-m \alpha^{2}\right)$ into Eq. (13), we find

$$
a_{B F}^{\text {Born }}=\frac{2 m_{B F} \sqrt{\left|\varepsilon_{b}\right|-m \alpha^{2}}}{m \sqrt{m}\left|\varepsilon_{b}\right|}
$$

in the Born approximation, suggesting that the fermion-dimer interaction is repulsive for all parameters. In Fig. 4, we show $a_{B F}^{\text {Born }}$ as a function of $1 /\left(m \alpha a_{s}\right)$, which is analytically tractable in three limits: (i) $a_{B F}^{\text {Born }}=-24 a_{s} / 7$ in the limit when $1 /\left(m \alpha a_{s}\right) \ll-1$, (ii) $a_{B F}^{\text {Born }}=6 \sqrt{2} /[m \alpha(5 \sqrt{2}-1)] \approx$ $1.40 /(m \alpha)$ in the unitarity limit when $1 /\left(m \alpha a_{s}\right)=0$, and (iii) $a_{B F}^{\mathrm{Born}}=8 a_{s} / 3$ in the limit when $1 /\left(m \alpha a_{s}\right) \gg 1$. Note that the latter limit recovers the usual three-body problem with no SOC in the $1 /\left(m \alpha a_{s}\right) \gg 1$ limit when $\alpha \rightarrow 0^{+}$.

To go beyond the Born approximation, we consider the one-loop contribution that is shown in Fig. 3(b). In accordance 


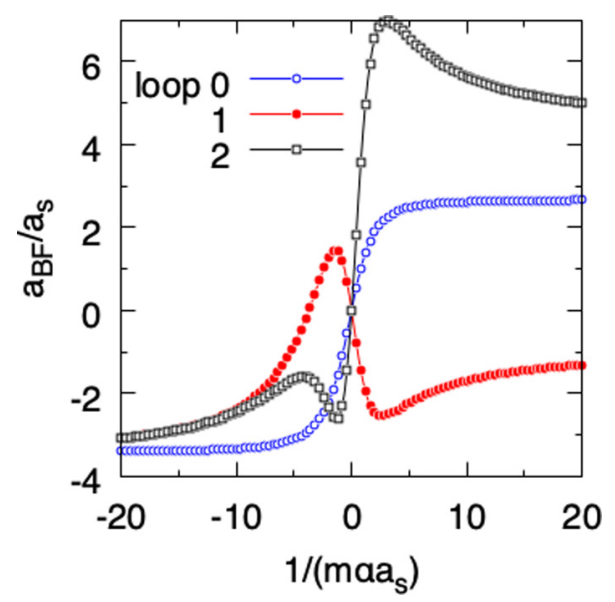

FIG. 4. Fermion-dimer scattering length in the zero-loop Born, one-loop, and two-loop approximations. The higher-loop contributions form a perturbative series in the $1 /\left(m \alpha a_{s}\right)<0$ region, but they are of similar order in the $1 /\left(m \alpha a_{s}\right)>0$ region.

with the Feynman rules, this diagram is given by

$$
\begin{aligned}
\mathcal{T}_{k}^{(1)}(0)= & \sum_{q} \sigma_{y} G^{\mathrm{T}}\left(\mathbf{q}-\mathbf{k}, q_{0}-k_{0}+\varepsilon_{b}\right) \sigma_{y} D\left(\mathbf{q}, q_{0}+\varepsilon_{b}\right) \\
& \times G\left(\mathbf{k}-\mathbf{q}, k_{0}-q_{0}\right) \sigma_{y} G^{\mathrm{T}}\left(\mathbf{q}-\mathbf{k}, q_{0}-k_{0}+\varepsilon_{b}\right) \sigma_{y} .
\end{aligned}
$$

Noting the relation $\left(\sigma_{0}+\widehat{\mathbf{A}} \cdot \boldsymbol{\sigma}\right)\left(\sigma_{0}+\widehat{\mathbf{B}} \cdot \boldsymbol{\sigma}\right)=(1+\widehat{\mathbf{A}}$. $\widehat{\mathbf{B}}) \sigma_{0}+(\widehat{\mathbf{A}}+\widehat{\mathbf{B}}+i \widehat{\mathbf{A}} \times \widehat{\mathbf{B}}) \cdot \boldsymbol{\sigma}$, we first integrate $q_{0}$ in the upper half plane in which there are two simple poles at $q_{0}=k_{0}-\varepsilon_{\mathbf{q}}^{ \pm}$, and we reduce the $t$-matrix contribution to

$$
\mathcal{T}_{k}^{(1)}(0)=\frac{1}{2} \sum_{s \mathbf{q}} \frac{D\left(\mathbf{q}+\mathbf{k}, k_{0}+\varepsilon_{b}-\varepsilon_{\mathbf{q}}^{s}\right)}{\left(2 \varepsilon_{\mathbf{q}}^{s}-\varepsilon_{b}\right)^{2}}\left(\sigma_{0}-s \widehat{\mathbf{q}} \cdot \boldsymbol{\sigma}\right) .
$$

Noting that $\mathcal{T}_{k}(0)$ has a spherical symmetry in $\mathbf{k}$ space, we choose an incoming momentum $\mathbf{k}=m \alpha \widehat{k}_{z}$ that is aligned with the $z$ axis, and we perform the remaining integrations numerically in the q space [28]. In Fig. 4, we show how the one-loop contribution affects $a_{B F}^{\text {Born }}$ as a function of $1 /\left(m \alpha a_{s}\right)$. In the one-loop approximation, we find that $a_{B F}^{\text {Born }}$ becomes attractive in the $1 /\left(m \alpha a_{s}\right)>0$ region, which is not physical.

To go further beyond the Born approximation, we next consider the two-loop contribution that is represented in Fig. 3(c). In accordance with the Feynman rules, this diagram is given by

$$
\begin{aligned}
\mathcal{T}_{k}^{(2)}(0)= & \sum_{q Q} \sigma_{y} G^{\mathrm{T}}\left(\mathbf{q}-\mathbf{k}, q_{0}-k_{0}+\varepsilon_{b}\right) \sigma_{y} D\left(\mathbf{q}, q_{0}+\varepsilon_{b}\right) \\
& \times G\left(\mathbf{k}-\mathbf{q}, k_{0}-q_{0}\right) \sigma_{y} G^{\mathrm{T}}\left(\mathbf{Q}-\mathbf{k}+\mathbf{q}, Q_{0}-k_{0}\right. \\
& \left.+q_{0}+\varepsilon_{b}\right) \sigma_{y} G\left(\mathbf{k}-\mathbf{Q}, k_{0}-Q_{0}\right) D\left(\mathbf{Q}, Q_{0}+\varepsilon_{b}\right) \sigma_{y} \\
& \times G^{\mathrm{T}}\left(\mathbf{Q}-\mathbf{k}, Q_{0}-k_{0}+\varepsilon_{b}\right) \sigma_{y},
\end{aligned}
$$

where a minus sign is included due to the fermion exchange. We integrate $q_{0}$ and $Q_{0}$ in their upper half planes in which there are two simple poles at $q_{0}=k_{0}-\varepsilon_{\mathbf{q}-\mathbf{k}}^{ \pm}$and two simple poles at $Q_{0}=k_{0}-\varepsilon_{\mathbf{Q}-\mathbf{k}}^{ \pm}$. In addition, by taking advantage of the symmetry of the diagram with respect to the internal variables $\mathbf{q}$ and $\mathbf{Q}$, we reduce the $t$-matrix contribution to

$$
\begin{aligned}
\mathcal{T}_{k}^{(2)}(0)= & \frac{1}{8} \sum_{s s^{\prime} s^{\prime \prime} \mathbf{q} \mathbf{Q}} \frac{D\left(\mathbf{q}+\mathbf{k}, k_{0}+\varepsilon_{b}-\varepsilon_{\mathbf{q}}^{s}\right) D\left(\mathbf{Q}+\mathbf{k}, k_{0}+\varepsilon_{b}-\varepsilon_{\mathbf{Q}}^{s^{\prime}}\right)}{\left(2 \varepsilon_{\mathbf{q}}^{s}-\varepsilon_{b}\right)\left(2 \varepsilon_{\mathbf{Q}}^{s^{\prime}}-\varepsilon_{b}\right)\left(\varepsilon_{\mathbf{q}}^{s}+\varepsilon_{\mathbf{Q}}^{s^{\prime}}+\varepsilon_{\mathbf{K}}^{s^{\prime \prime}}-k_{0}-\varepsilon_{b}\right)}\left(\left\{1+s s^{\prime \prime} \widehat{\mathbf{q}} \cdot \widehat{\mathbf{K}}+s^{\prime} s^{\prime \prime} \widehat{\mathbf{Q}} \cdot \widehat{\mathbf{K}}+s s^{\prime} \widehat{\mathbf{q}} \cdot \widehat{\mathbf{Q}}\right\} \sigma_{0}\right. \\
& \left.\left.+\left\{s \mathbf{q}+s^{\prime} \widehat{\mathbf{Q}}+s^{\prime \prime} \widehat{\mathbf{K}}-s s^{\prime} s^{\prime \prime}[(\widehat{\mathbf{q}} \cdot \widehat{\mathbf{Q}}) \widehat{\mathbf{K}}-(\widehat{\mathbf{Q}} \cdot \widehat{\mathbf{K}}) \widehat{\mathbf{q}} / 2-(\widehat{\mathbf{q}} \cdot \widehat{\mathbf{K}}) \widehat{\mathbf{Q}} / 2)\right]\right\} \cdot \boldsymbol{\sigma}\right),
\end{aligned}
$$

where $\mathbf{K}=\mathbf{Q}+\mathbf{q}+\mathbf{k}$. We again choose an incoming momentum $\mathbf{k}=m \alpha k_{z}$ that is aligned with the $z$ axis, and we perform the remaining integrations numerically in the $\mathbf{q}$ and $\mathbf{Q}$ spaces [28]. In Fig. 4, we show how the combination of the one-loop and two-loop contributions affects $a_{B F}^{\text {Born }}$ as a function of $1 /\left(m \alpha a_{s}\right)$. While the two-loop contribution is negligible in the $1 /\left(m \alpha a_{s}\right) \ll-1$ limit, it leads to a repulsive $a_{B F}$ in the $1 /\left(m \alpha a_{s}\right)>0$ region.

By comparing the zero-loop, one-loop, and two-loop approximations in Fig. 4, we observe that while the higher-loop contributions form a perturbative series in the $1 /\left(m \alpha a_{s}\right)<0$ region, they are of similar order in the $1 /\left(m \alpha a_{s}\right)>0$ region. Noting that $Z$ decays to 0 in the $1 /\left(m \alpha a_{s}\right) \rightarrow-\infty$ limit, and that it increases as $\sqrt{\left|\varepsilon_{b}\right|}$ in the $1 /\left(m \alpha a_{s}\right) \rightarrow+\infty$ limit, this observation is caused by the incremental growth of the power of $Z$ that is coming from the additional dimer propagators within each loop. For this reason, a proper description of the latter region requires infinitely many loop diagrams at all orders $[1-7,13]$. A practical way to handle such summations is presented in Fig. 5, where the fermion-dimer scattering $t$ matrix is determined by repeating the fermion-exchange process infinitely many times, forming eventually an integral equation. In accordance with the Feynman rules, this diagram

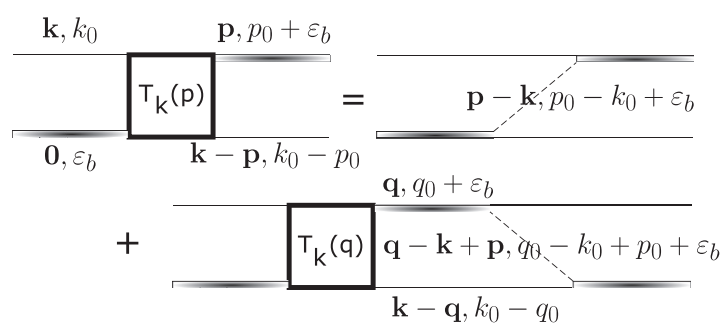

FIG. 5. Diagrammatic representation of the three-body problem. The fermion-dimer scattering $t$ matrix is determined by repeating the fermion-exchange process infinitely many times, forming eventually an integral equation. 
is given by

$$
\begin{aligned}
\mathcal{T}_{k}(p)= & -\sigma_{y} G^{\mathrm{T}}\left(\mathbf{p}-\mathbf{k}, p_{0}-k_{0}+\varepsilon_{b}\right) \sigma_{y} \\
& -\sum_{q} \mathcal{T}_{k}(q) D\left(\mathbf{q}, q_{0}+\varepsilon_{b}\right) G\left(\mathbf{k}-\mathbf{q}, k_{0}-q_{0}\right) \\
& \times \sigma_{y} G^{\mathrm{T}}\left(\mathbf{q}-\mathbf{k}+\mathbf{p}, q_{0}-k_{0}+p_{0}+\varepsilon_{b}\right) \sigma_{y},
\end{aligned}
$$

where the minus signs are due to the fermion exchanges. Integrating $q_{0}$ in the upper half plane where $\mathcal{T}_{k}(q)$ is analytic and there are two simple poles at $q_{0}=k_{0}-\varepsilon_{\mathbf{q}-\mathbf{k}}^{ \pm}$, we reduce the $t$-matrix equation to

$$
\begin{aligned}
\mathcal{T}_{k}\left(\mathbf{p}, p_{0}\right)= & -\frac{1}{2} \sum_{s} \frac{\sigma_{0}-s \widehat{\mathbf{k}}^{\prime} \cdot \boldsymbol{\sigma}}{p_{0}-k_{0}+\varepsilon_{b}-\varepsilon_{\mathbf{k}^{\prime}}^{s}} \\
& -\frac{1}{4} \sum_{s s^{\prime} \mathbf{q}} \frac{D\left(\mathbf{q}, k_{0}+\varepsilon_{b}-\varepsilon_{\mathbf{Q}}^{s}\right)}{p_{0}+\varepsilon_{b}-\varepsilon_{\mathbf{Q}}^{s}-\varepsilon_{\mathbf{K}}^{s^{\prime}}} \mathcal{T}_{k}\left(\mathbf{q}, k_{0}-\varepsilon_{\mathbf{Q}}^{s}\right) \\
& \times\left[\left(1-s s^{\prime} \widehat{\mathbf{Q}} \cdot \widehat{\mathbf{K}}\right) \sigma_{0}+\left(s \widehat{\mathbf{Q}}-s^{\prime} \widehat{\mathbf{K}}\right.\right. \\
& \left.\left.-i s s^{\prime} \widehat{\mathbf{Q}} \times \widehat{\mathbf{K}}\right) \cdot \boldsymbol{\sigma}\right] .
\end{aligned}
$$

Here $\mathbf{k}^{\prime}=\mathbf{p}-\mathbf{k}, \mathbf{Q}=\mathbf{k}-\mathbf{q}$, and $\mathbf{K}=\mathbf{p}-\mathbf{k}+\mathbf{q}$ are introduced for the simplicity of the presentation.

In the usual three-body problem with no SOC, $t_{0}\left(\mathbf{p}, p_{0}\right)$ is not only a real function but it is also restricted to the socalled on-the-shell value $t_{0}\left[\mathbf{p}, p_{0}=-p^{2} /(2 m)\right]$ for both the incoming and outgoing fermions [1-7,13]. In addition, using the spherical symmetry of the $t$ matrix, the problem reduces to a simple integral equation with a single variable for $t_{0}(|\mathbf{p}|)$, whose numerical computation converges very fast. However, since the helicity bands are coupled due to the nonstationary dimers, there are two shells contributing to Eq. (22). Furthermore, given that the $t$ matrix is a $2 \times 2$ matrix with complex elements, this reduces Eq. (22) to eight coupled integral equations. Unfortunately, this is quite complicated, and the exact numerical solution of the three-body problem remains an open problem.

\section{FOUR-BODY PROBLEM}

Motivated by the overall success of the Born approximation in the fermion-dimer scattering problem, here we apply the diagrammatic approach to the scattering $t$ matrix $t_{0}^{B B}(0)$ between two stationary dimers in the one-loop Born and two-loop approximations. Despite its simplicity, we expect $a_{B B}^{\text {Born }}$ to be quite accurate in the $1 /\left(m \alpha a_{s}\right) \ll-1$ limit as the higher-order contributions form a perturbative series in the $1 /\left(m \alpha a_{s}\right)<0$ region. However, our results are only qualitative in the $1 /\left(m \alpha a_{s}\right)>0$ region, whose accurate description is beyond the scope of this paper [2,3,9].

The diagrammatic representation of the Born contribution to the dimer-dimer scattering $t$ matrix is shown in Fig. 6(a) $[2,3,8,9]$. In accordance with the Feynman rules, it is given by

$$
t_{0}^{B B(1)}(0)=-\frac{\operatorname{Tr}}{2} \sum_{k}\left[\sigma_{y} G\left(\mathbf{k}, k_{0}+\varepsilon_{b}\right) \sigma_{y} G^{\mathrm{T}}\left(-\mathbf{k},-k_{0}\right)\right]^{2},
$$

where the minus sign is due to the fermion exchange. Noting the relations $\left(\sigma_{0} \pm \widehat{\mathbf{A}} \cdot \boldsymbol{\sigma}\right)\left(\sigma_{0} \pm \widehat{\mathbf{A}} \cdot \boldsymbol{\sigma}\right)=2\left(\sigma_{0} \pm \hat{\mathbf{A}} \cdot \boldsymbol{\sigma}\right)$,

(a)

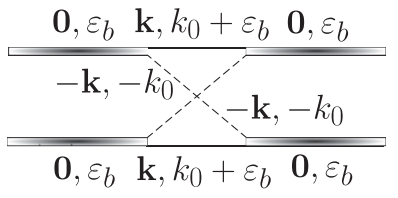

(b)

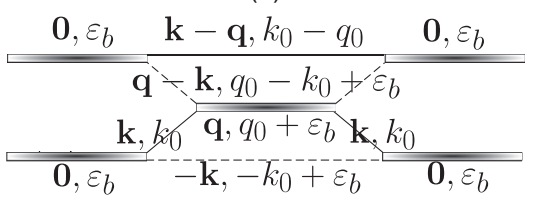

FIG. 6. Diagrammatic representation of the (a) one-loop Born and (b) two-loop contributions to the dimer-dimer scattering $t$ matrix.

and $\left(\sigma_{0} \pm \widehat{\mathbf{A}} \cdot \boldsymbol{\sigma}\right)\left(\sigma_{0} \mp \widehat{\mathbf{A}} \cdot \boldsymbol{\sigma}\right)=0$, we first integrate $k_{0}$ in the upper half plane in which there are two double poles at $k_{0}=-\varepsilon_{\mathbf{k}}^{ \pm}$, and we reduce the $t$-matrix contribution to

$$
t_{0}^{B B^{(1)}}(0)=\frac{1}{2} \sum_{s \mathbf{k}} \frac{2}{\left(2 \varepsilon_{\mathbf{k}}^{s}-\varepsilon_{b}\right)^{3}} .
$$

This is a physically intuitive result because, since all dimers are stationary in the Born diagram, the helicity bands are not coupled, and the diagram can be directly expressed as $t_{0}^{B B^{(1)}}(0)=(-1 / 2) \sum_{s k}\left[G_{s}\left(\mathbf{k}, k_{0}+\varepsilon_{b}\right) G_{s}\left(-\mathbf{k},-k_{0}\right)\right]^{2}$.

In this paper, we are interested in the dimer-dimer scattering length $a_{B B}$ that is determined by $[2,3,8,9]$

$$
a_{B B}=\frac{m_{B}}{4 \pi} Z^{2} t_{0}^{B B}(0) \text {. }
$$

Plugging $t_{0}^{B B^{(1)}}(0)=m \sqrt{m}\left(\left|\varepsilon_{b}\right|+2 m \alpha^{2}\right) /\left[16 \pi\left(\left|\varepsilon_{b}\right|-m \alpha^{2}\right)^{5 / 2}\right]$ into the above equation, we find [25-27]

$$
a_{B B}^{\text {Born }}=\frac{m_{B}\left(\left|\varepsilon_{b}\right|+2 m \alpha^{2}\right) \sqrt{\left|\varepsilon_{b}\right|-m \alpha^{2}}}{m \sqrt{m}\left|\varepsilon_{b}\right|^{2}},
$$

in the Born approximation, which suggests that the dimerdimer interaction is repulsive for all parameters. In Fig. 7, we show $a_{B B}^{\text {Born }}$ as a function of $1 /\left(m \alpha a_{s}\right)$, which is analytically tractable in three limits: (i) $a_{B B}^{\text {Born }}=-18 a_{s}$ in the limit when $1 /\left(m \alpha a_{s}\right) \ll-1$, (ii) $a_{B B}^{\text {Born }}=3 \sqrt{2} /[m \alpha(2 \sqrt{2}-1)] \approx$ $2.32 /(m \alpha)$ in the unitarity limit when $1 /\left(m \alpha a_{s}\right)=0$, and (iii) $a_{B B}^{\text {Born }}=2 a_{s}$ in the limit when $1 /\left(m \alpha a_{s}\right) \gg 1$. Note that the latter limit recovers the usual four-body problem with no SOC in the $1 /\left(m \alpha a_{s}\right) \gg 1$ limit when $\alpha \rightarrow 0^{+}$.

To go beyond the Born approximation, we consider the two-loop contribution that is shown in Fig. 6(b). In accordance with the Feynman rules, this diagram is given by

$$
\begin{aligned}
t_{0}^{B B^{(2)}}(0)= & -\frac{\operatorname{Tr}}{2} \sum_{k q} \sigma_{y} G^{\mathrm{T}}\left(\mathbf{q}-\mathbf{k}, q_{0}-k_{0}+\varepsilon_{b}\right) \sigma_{y} \\
& \times G\left(\mathbf{k}, k_{0}\right) D\left(\mathbf{q}, q_{0}+\varepsilon_{b}\right) \sigma_{y} G^{\mathrm{T}}\left(-\mathbf{k},-k_{0}+\varepsilon_{b}\right) \\
& \times \sigma_{y} G\left(\mathbf{k}, k_{0}\right) \sigma_{y} G^{\mathrm{T}}\left(\mathbf{q}-\mathbf{k}, q_{0}-k_{0}+\varepsilon_{b}\right) \\
& \times \sigma_{y} G\left(\mathbf{k}-\mathbf{q}, k_{0}-q_{0}\right) .
\end{aligned}
$$

We first integrate $q_{0}$ in the upper half plane in which there are two simple poles at $q_{0}=k_{0}-\varepsilon_{\mathbf{q}-\mathbf{k}}^{ \pm}$, and then we integrate $k_{0}$ in the upper half plane in which there are two simple poles at 


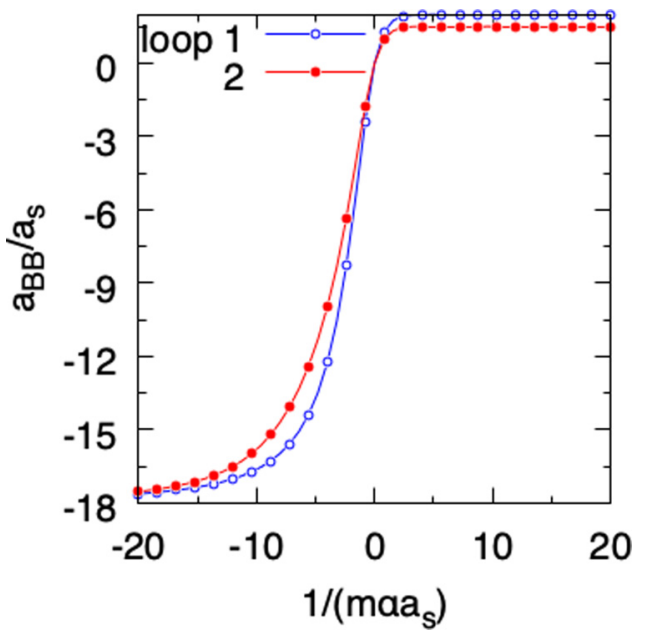

FIG. 7. Dimer-dimer scattering length in the one-loop Born and two-loop approximations. The higher-loop contributions form a perturbative series in the $1 /\left(m \alpha a_{s}\right)<0$ region, but they are of similar order in the $1 /\left(m \alpha a_{s}\right)>0$ region.

$k_{0}=\varepsilon_{b}-\varepsilon_{\mathbf{k}}^{ \pm}$. This reduces the $t$-matrix contribution to

$$
t_{0}^{B B^{(2)}}(0)=\frac{1}{4} \sum_{s s^{\prime} \mathbf{k} \mathbf{q}} \frac{D\left(\mathbf{q}, 2 \varepsilon_{b}-\varepsilon_{\mathbf{k}}^{s}-\varepsilon_{\mathbf{Q}}^{s^{\prime}}\right)}{\left(2 \varepsilon_{\mathbf{k}}^{s}-\varepsilon_{b}\right)^{2}\left(2 \varepsilon_{\mathbf{Q}}^{s^{\prime}}-\varepsilon_{b}\right)^{2}}\left(1+s s^{\prime} \widehat{\mathbf{k}} \cdot \widehat{\mathbf{Q}}\right),
$$

where $\mathbf{Q}=\mathbf{k}+\mathbf{q}$, and the remaining integrations are performed numerically in the $\mathbf{k}$ and $\mathbf{q}$ spaces [28]. In Fig. 7, we show how the two-loop contribution affects $a_{B B}^{\text {Born }}$ as a function of $1 /\left(m \alpha a_{s}\right)$. While the two-loop contribution is negligible in the $1 /\left(m \alpha a_{s}\right) \ll-1$ limit, it is comparable to $a_{B B}^{\text {Born }}$ in the $1 /\left(m \alpha a_{s}\right)>0$ region.

\section{MANY-BODY PROBLEM}

The fermion-dimer and dimer-dimer scattering lengths offer valuable insights for some of the many-body properties of Fermi gases. For instance, in the case of populationimbalanced Fermi gases, $a_{B F}$ and $a_{B B}$ can be used to map the strongly interacting Fermi-Fermi mixture of $\uparrow$ and $\downarrow$ fermions to a weakly interacting Bose-Fermi mixture of paired fermions (dimers) and unpaired (excess) ones $[4,15,16]$. In the parameter regime where this effective description holds, the existing literature on true Bose-Fermi mixtures can be easily utilized to characterize the imbalanced Fermi gases.

For instance, it is well-known that a weakly interacting Bose-Fermi mixture is unstable against phase separation with a negative compressibility when the density of fermions $n_{F}$ satisfies [22] $n_{F} \geqslant 4 \pi^{4} U_{B B}^{3} /\left(3 m_{F}^{3} U_{B F}^{6}\right)$ in three dimensions, where $U_{B B}=4 \pi a_{B B} / m_{B}$ is the repulsive interaction between bosons, and $U_{B F}=4 \pi a_{B F} / m_{B F}$ is the repulsive interaction between fermions and bosons. Thus, the Bose-Fermi mixture phase separates when

$$
n_{F} \geqslant \frac{4 \pi}{3} \frac{m^{3} m_{B}^{3}}{\left(m_{B}+m\right)^{6}} \frac{a_{B B}^{3}}{a_{B F}^{6}},
$$

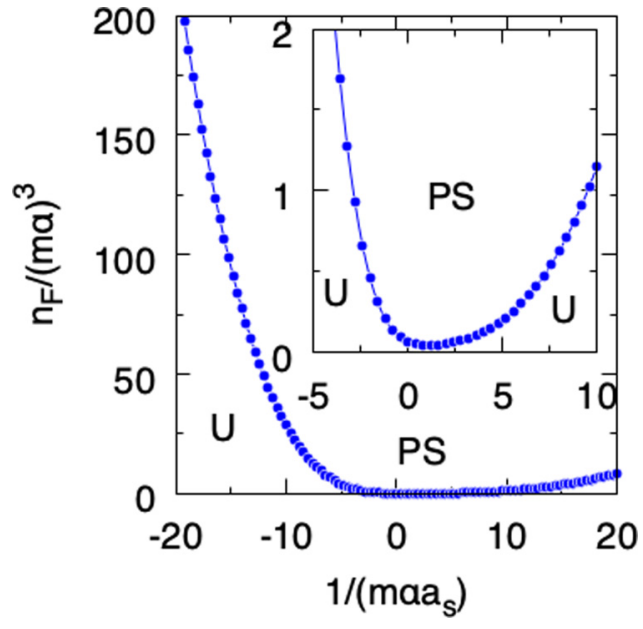

FIG. 8. Critical boundary between the uniform superfluid (U) and the phase separation (PS) that is determined by the effective weakly interacting Bose-Fermi mixture description of a populationimbalanced Fermi gas in the Born approximation.

and it is otherwise uniform. By plugging the Born approximations Eqs. (16) and (26) into Eq. (29), we obtain the corresponding relation for the stability of a populationimbalanced Fermi gas with SOC. The critical boundary between the uniform superfluid and the phase separation is shown in Fig. 8.

Here we remark in passing that one can study BCS-BEC evolution for any given $a_{s}$ by tuning the strength $\alpha$ of the SOC, no matter how small or large the value of $a_{s}$ is and independently of its sign. Its physical mechanism is the SOC-induced enhancement of $\varepsilon_{b}$ through the increase of the single-particle density of states. In particular, when $\alpha$ is large, the nature of the bosons that make up the BEC is determined solely by $\alpha a_{s}$. For this reason, these bosons are sometimes called rashbons in the recent literature since their properties are determined by SOC alone. See Refs. [21-27] for further discussion, including the effective Gross-Pitaevskii description of the weakly interacting dimers in the BEC limit.

\section{ANISOTROPIC (RASHBA) SPIN-ORBIT COUPLING}

Our results can be easily generalized to anisotropic SOC fields. For instance, in the presence of a Rashba SOC, the one-body Hamiltonian is governed by $h_{\mathbf{k}}=\varepsilon_{\mathbf{k}} \sigma_{0}+\alpha \mathbf{k}_{\perp}$. $\boldsymbol{\sigma}$, where $\mathbf{k}=\left(\mathbf{k}_{\perp}, k_{z}\right)$ and $\mathbf{k}_{\perp}=\left(k_{x}, k_{y}\right)$, leading to $\varepsilon_{\mathbf{k}}^{s}=$ $\left(k_{\perp}^{2}+k_{z}^{2}\right) /(2 m)+s \alpha k_{\perp}$. Therefore, the ground state of the --helicity band corresponds to a degenerate ring of $\mathbf{k}_{\perp}$ states with the radius $k_{m}=m \alpha$ at $k_{z}=0$ and the energy $\varepsilon_{\mathbf{k}_{m}}^{-}=$ $-m \alpha^{2} / 2$.

In the lowest order in $\mathbf{q}=\left(\mathbf{q}_{\perp}, q_{z}\right)$ and $q_{0}$, Eq. (6) has the generic structure of a simple pole [22-27]:

$$
D\left(\mathbf{q}, q_{0}\right)=\frac{Z}{q_{0}-q_{\perp}^{2} /\left(2 m_{B, \perp}\right)-q_{z}^{2} /\left(2 m_{B, z}\right)+\mu_{B}+i 0^{+}},
$$

where $Z=8 \pi\left(\left|\varepsilon_{b}\right|-m \alpha^{2}\right) /\left(m \sqrt{m\left|\varepsilon_{b}\right|}\right)$ corresponds to the residue of the pole, and $2 m / m_{B, \perp}=\left(2\left|\varepsilon_{b}\right|-m \alpha^{2}\right) /\left(2\left|\varepsilon_{b}\right|\right)-$ 
Isotropic

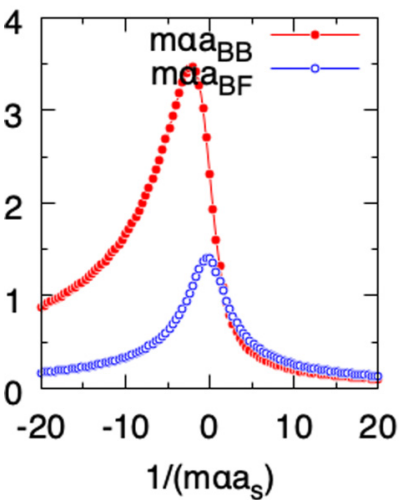

Rashba

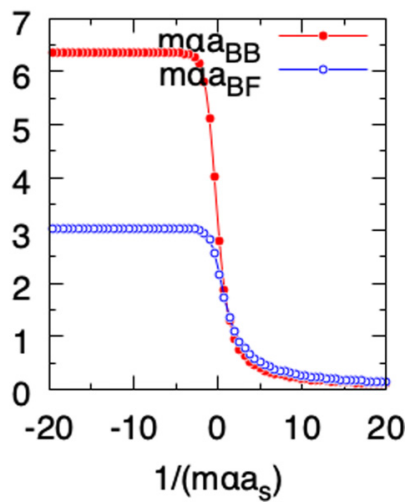

FIG. 9. Dimer-dimer (red) and fermion-dimer (blue) scattering lengths for the isotropic SOC (left: same as in Fig. 4) versus the Rashba SOC (right) in the Born approximations.

$\left[\left(\left|\varepsilon_{b}\right|-m \alpha^{2}\right) /\left(2\left|\varepsilon_{b}\right|\right)\right] \log \left(1-m \alpha^{2} /\left|\varepsilon_{b}\right|\right)$ and $m_{B, z}=2 m$ determine the anisotropic effective mass of the dimer. In addition, $\mu_{B}=\left|\varepsilon_{b}\right|$ is determined by $1 /\left(m \alpha a_{s}\right)=\sqrt{\left|\varepsilon_{b}\right| /\left(m \alpha^{2}\right)}-$ $\log \left[\sqrt{\left|\varepsilon_{b}\right| /\left(\left|\varepsilon_{b}\right|-m \alpha^{2}\right)}+\sqrt{m \alpha^{2} /\left(\left|\varepsilon_{b}\right|-m \alpha^{2}\right)}\right]$. This expression is analytically tractable in three limits [21-23]: we find that (i) $\varepsilon_{b}=-m \alpha^{2}-4 m \alpha^{2} e^{2 /\left(m \alpha a_{s}\right)-2}$ and $m_{B, \perp}=4 m$ in the limit when $1 /\left(m \alpha a_{s}\right) \ll-1$, (ii) $\varepsilon_{b} \approx-1.44 m \alpha^{2}$ and $m_{B, \perp} \approx$ $2.40 m$ in the unitarity limit when $1 /\left(m \alpha a_{s}\right)=0$, and (iii) $\varepsilon_{b}=$ $-1 /\left(m a_{s}^{2}\right)$ and $m_{B, \perp}=2 m$ in the limit when $1 /\left(m \alpha a_{s}\right) \gg 1$. Note that the latter limit recovers the usual two-body problem with no SOC in the $1 /\left(m \alpha a_{s}\right) \gg 1$ limit when $\alpha \rightarrow 0^{+}$.

Since $k=\left(m \alpha \widehat{\mathbf{k}_{\perp}},-m \alpha^{2} / 2\right)$ corresponds to the lowestenergy eigenstate in the --helicity band, Eq. (15) gives $t_{k}^{--(0)}(0)=1 /\left(\left|\varepsilon_{b}\right|-m \alpha^{2}\right)$. By plugging it into Eq. (13), we find

$$
a_{B F}^{\text {Born }}=\frac{2 m_{B F}}{m \sqrt{m\left|\varepsilon_{b}\right|}}
$$

in the Born approximation, where $m_{B}$ refers to the geometric mean $\left(m_{B, \perp}^{2} m_{B, z}\right)^{1 / 3}$ of the anisotropic effective mass [27]. In Fig. 9, we show $a_{B F}^{\text {Born }}$ as a function of $1 /\left(m \alpha a_{s}\right)$, which is analytically tractable in three limits: (i) $a_{B F}^{\text {Born }} \approx 3.04 /(m \alpha)$ in the limit when $1 /\left(m \alpha a_{s}\right) \ll-1$, (ii) $a_{B F}^{\text {Born }} \approx 2.31 /(m \alpha)$ in the unitarity limit when $1 /\left(m \alpha a_{s}\right)=0$, and (iii) $a_{B F}^{\text {Born }}=8 a_{s} / 3$ in the limit when $1 /\left(m \alpha a_{s}\right) \gg 1$. Note again the latter limit recovers the usual three-body problem with no SOC in the $1 /\left(m \alpha a_{s}\right) \gg 1$ limit when $\alpha \rightarrow 0^{+}$.

Similarly, Eq. (24) gives $t_{0}^{B B^{(1)}}(0)=m \sqrt{m}\left(\left|\varepsilon_{b}\right|+m \alpha^{2}\right) /$ $\left[16 \pi\left(\left|\varepsilon_{b}\right|-m \alpha^{2}\right)^{2} \sqrt{\left|\varepsilon_{b}\right|}\right]$, and by plugging it in Eq. (25), we find

$$
a_{B B}^{\mathrm{Born}}=\frac{m_{B}\left(\left|\varepsilon_{b}\right|+m \alpha^{2}\right)}{m \sqrt{m} \sqrt{\left|\varepsilon_{b}\right|^{3}}},
$$

in the Born approximation. In Fig. 9, we show $a_{B B}^{\text {Born }}$ as a function of $1 /\left(m \alpha a_{s}\right)$, which is analytically tractable in three limits: (i) $a_{B B}^{\text {Born }} \approx 6.35 /(m \alpha)$ in the limit when $1 /\left(m \alpha a_{s}\right) \ll-1$, (ii) $a_{B B}^{\mathrm{B} o r n} \approx 3.19 /(m \alpha)$ in the unitarity limit when $1 /\left(m \alpha a_{s}\right)=0$, and (iii) $a_{B B}^{\text {Born }}=2 a_{s}$ in the limit when $1 /\left(m \alpha a_{s}\right) \gg 1$. Note again that the latter limit recovers the usual four-body problem with no SOC in the $1 /\left(m \alpha a_{s}\right) \gg 1$ limit when $\alpha \rightarrow 0^{+}$.

In contrast to the isotropic SOC case where $a_{B F}$ and $a_{B B}$ are nonmonotonous functions of $1 /\left(m \alpha a_{s}\right)$, they evolve monotonously in the Rashba SOC. Their saturations in the $1 /\left(m \alpha a_{s}\right) \ll-1$ limit are caused by the exact cancellation of the decay of $Z$ with the divergence of the $t$ matrices. The decay is faster in the isotropic case, causing the peak in the intermediate region. Despite this major difference, the isotropic and Rashba SOC cases share some common properties. For instance, the decrease, increase, and saturation of $a_{B F}$ are in full coordination with those of $a_{B B}$. In addition, we note that $a_{B F}$ is greater (smaller) than $a_{B B}$ in approximately the $1 /\left(m \alpha a_{s}\right) \gtrless 0$ regions.

\section{CONCLUSION}

In summary, we studied how SOC affects the fermiondimer and dimer-dimer scattering lengths in the Born approximation, and we benchmarked their accuracy with the higher-order approximations. We considered both isotropic and Rashba couplings in three dimensions and found that the Born approximation gives accurate results for both $a_{B F}$ and $a_{B B}$ in the $1 /\left(m \alpha a_{s}\right) \ll-1$ limit. This is because while the higher-loop contributions form a perturbative series in the $1 /\left(m \alpha a_{s}\right)<0$ region, they are of similar order in the $1 /\left(m \alpha a_{s}\right)>0$ region. We found that the perturbations are controlled by the residue $Z$ of the dimer propagator, which decays to 0 in the $1 /\left(m \alpha a_{s}\right) \rightarrow-\infty$ limit and increases as $\sqrt{\left|\varepsilon_{b}\right|}$ in the $1 /\left(m \alpha a_{s}\right)>0$ region. Therefore, it may be sufficient to consider a finite number of higher-order loop diagrams in the $1 /\left(m \alpha a_{s}\right)<0$ region.

On the other hand, a proper description of the $1 /\left(m \alpha a_{s}\right)>$ 0 region requires infinitely many loop diagrams at all orders. In the case of the three-body problem, we derived a coupled set of integral equations for the exact atom-dimer scattering length, but its numerical solutions remain an open problem. It may be possible to solve the exact three-body problem
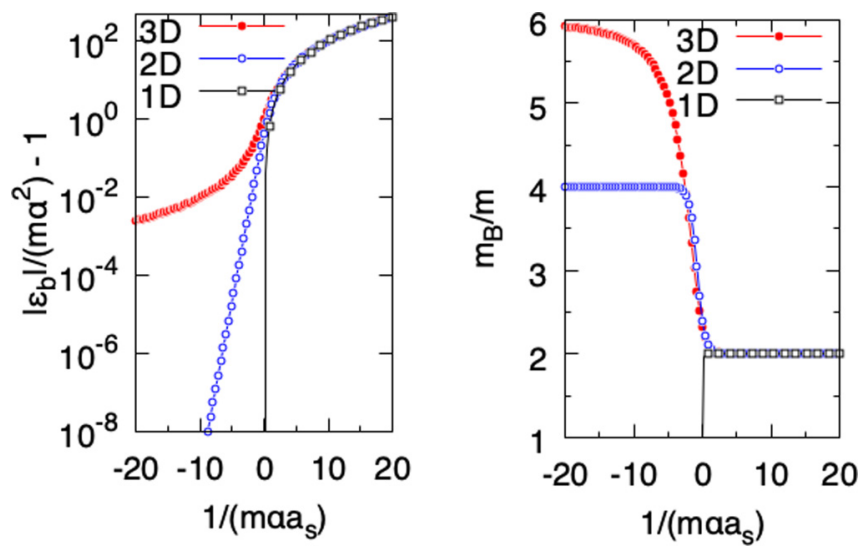

FIG. 10. Binding energy $\left|\varepsilon_{b}\right|-m \alpha^{2}$ and effective mass $m_{B, x}$ of the dimer. Here, the 3D SOC field is shown in red and refers to $\alpha \mathbf{k}$ with $\mathbf{k}=\left(k_{x}, k_{y}, k_{z}\right)$, the 2D field is shown in blue and refers to $\alpha \mathbf{k}_{\perp}$ with $\mathbf{k}_{\perp}=\left(k_{x}, k_{y}, 0\right)$, and the $1 \mathrm{D}$ field is shown in black and refers to $\mathbf{k}=\left(k_{x}, 0,0\right)$. 
through partial-wave expansion and address the possibility of a three-body bound state in this system. In addition, one may also study the importance of the full momentum and/or full frequency dependencies of the dimer propagator in the threeand/or four-body problems.

\section{ACKNOWLEDGMENT}

The author acknowledges funding from TÜBITAK, Grant No. 11001-118F359.

\section{APPENDIX: BINDING ENERGY AND EFFECTIVE MASS OF THE DIMER}

For the sake of completeness, we present the binding energy $\left|\varepsilon_{b}\right|-m \alpha^{2}$ and the effective mass $m_{B}$ of the dimer in
Fig. 10, where the three-dimensional (3D) SOC field refers to $\alpha \mathbf{k}$ with $\mathbf{k}=\left(k_{x}, k_{y}, k_{z}\right)$, the 2D field refers to $\alpha \mathbf{k}_{\perp}$ with $\mathbf{k}_{\perp}=\left(k_{x}, k_{y}, 0\right)$, and the $1 \mathrm{D}$ field refers to $\mathbf{k}=\left(k_{x}, 0,0\right)$. The latter case is trivial because as the 1D SOC field can be gauged away from the $\mathbf{k}$-space integrations, it is equivalent to the usual two-body problem with no SOC [22]. Therefore, a twobody bound state exists only when $a_{s}>0$ with an effective mass $m_{B}=2 m$ that is isotropic in space.

In contrast to the $1 \mathrm{D}$ case, a two-body bound state exists for all $a_{s}$ in both 3D (isotropic) and 2D (Rashba) SOC fields, which is caused by the increase in the low-energy density of one-body states [21-27]. In addition, while the effective mass of the dimer is isotropic in the $3 \mathrm{D}$ case where $m_{B}=m_{B, x}=$ $m_{B, y}=m_{B, z}$ is shown in the figure, it is anisotropic in the $2 \mathrm{D}$ case where only $m_{B, \perp}=m_{B, x}=m_{B, y}$ is shown in the figure and $m_{B, z}=2 m$ for all $a_{s}$.
[1] P. F. Bedaque and U. van Kolck, Nucleon-deuteron scattering from an effective field theory, Phys. Lett. B 428, 221 (1998).

[2] I. V. Brodsky, M. Yu. Kagan, A. V. Klaptsov, R. Combescot, and X. Leyronas, Exact diagrammatic approach for dimer-dimer scattering and bound states of three and four resonantly interacting particles, JETP Lett. 82, 273 (2005); Phys. Rev. A 73, 032724 (2006).

[3] J. Levinsen and V. Gurarie, Properties of strongly paired fermionic condensates, Phys. Rev. A 73, 053607 (2006).

[4] M. Iskin and C. A. R. Sá de Melo, Fermi-Fermi mixtures in the strong-attraction limit, Phys. Rev. A 77, 013625 (2008).

[5] M. Iskin, Dimer-atom scattering between two identical fermions and a third particle, Phys. Rev. A 81, 043634 (2010).

[6] F. Alzetto, R. Combescot, and X. Leyronas, Atom-dimer scattering length for fermions with different masses: Analytical study of limiting cases, Phys. Rev. A 82, 062706 (2010).

[7] J. Levinsen and D. S. Petrov, Atom-dimer and dimer-dimer scattering in fermionic mixtures near a narrow Feshbach resonance, Eur. Phys. J. D 65, 67 (2011).

[8] P. Pieri and G. C. Strinati, Strong-coupling limit in the evolution from BCS superconductivity to Bose-Einstein condensation, Phys. Rev. B 61, 15370 (2000).

[9] F. Alzetto, R. Combescot, and X. Leyronas, Dimer-dimer scattering length for fermions with different masses: Analytical study for large mass ratio, Phys. Rev. A 87, 022704 (2013).

[10] G. V. Skorniakov and K. A. Ter-Martirosian, Three body problem for short range forces. I. Scattering of low energy neutrons by deuterons, Zh. Eksp. Teor. Fiz. 31, 775 (1956) [Sov. Phys. JETP 4, 648 (1957)].

[11] D. S. Petrov, Three-body problem in Fermi gases with shortrange interparticle interaction, Phys. Rev. A 67, 010703(R) (2003).

[12] D. S. Petrov, C. Salomon, and G. V. Shlyapnikov, Diatomic molecules in ultracold Fermi gases-novel composite bosons, J. Phys. B: At. Mol. Opt. Phys. 38, S645 (2005).

[13] R. Combescot, Three-Body Coulomb Problem, Phys. Rev. X 7, 041035 (2017).

[14] For example, see one of the most recent reviews by G. C. Strinati, P. Pieri, G. Roepke, P. Schuck, and M. Urban, The
BCS-BEC crossover: From ultra-cold Fermi gases to nuclear systems, Phys. Rep. 738, 1 (2018).

[15] P. Pieri and G. C. Strinati, Trapped Fermions with Density Imbalance in the Bose-Einstein Condensate Limit, Phys. Rev. Lett. 96, 150404 (2006).

[16] E. Taylor, A. Griffin, and Y. Ohashi, Spin-polarized Fermi superfluids as Bose-Fermi mixtures, Phys. Rev. A 76, 023614 (2007).

[17] L. W. Cheuk, A. T. Sommer, Z. Hadzibabic, T. Yefsah, W. S. Bakr, and M. W. Zwierlein, Spin-Injection Spectroscopy of a Spin-Orbit Coupled Fermi Gas, Phys. Rev. Lett. 109, 095302 (2012).

[18] R. A. Williams, M. C. Beeler, L. J. LeBlanc, K. Jiménez-García, and I. B. Spielman, Raman-Induced Interactions in a SingleComponent Fermi Gas Near an $s$-Wave Feshbach Resonance, Phys. Rev. Lett. 111, 095301 (2013).

[19] L. Huang, Z. Meng, P. Wang, P. Peng, S.-L. Zhang, L. Chen, D. Li, Q. Zhou, and J. Zhang, Experimental realization of a twodimensional synthetic spin-orbit coupling in ultracold Fermi gases, Nat. Phys. 12, 540 (2016).

[20] Z. Meng, L. Huang, P. Peng, D. Li, L. Chen, Y. Xu, C. Zhang, P. Wang, and J. Zhang, Experimental Observation of a Topological Band Gap Opening in Ultracold Fermi Gases with Two-Dimensional Spin-Orbit Coupling, Phys. Rev. Lett. 117, 235304 (2016).

[21] Z.-Q. Yu and H. Zhai, Spin-Orbit Coupled Fermi Gases across a Feshbach Resonance, Phys. Rev. Lett. 107, 195305 (2011).

[22] M. Iskin and A. L. Subaş1, Quantum phases of atomic Fermi gases with anisotropic spin-orbit coupling, Phys. Rev. A 84, 043621 (2011).

[23] H. Hu, L. Jiang, X.-J. Liu, and H. Pu, Probing Anisotropic Superfluidity in Atomic Fermi Gases with Rashba Spin-Orbit Coupling, Phys. Rev. Lett. 107, 195304 (2011).

[24] L. He and X.-G. Huang, BCS-BEC Crossover in 2D Fermi Gases with Rashba Spin-Orbit Coupling, Phys. Rev. Lett. 108, 145302 (2012).

[25] L. He and X.-G. Huang, BCS-BEC crossover in threedimensional Fermi gases with spherical spin-orbit coupling, Phys. Rev. B 86, 014511 (2012). 
[26] J. P. Vyasanakere and V. B. Shenoy, Rashbons: Properties and their significance, New J. Phys. 14, 043041 (2012).

[27] J. P. Vyasanakere and V. B. Shenoy, Collective excitations, emergent Galilean invariance, and boson-boson interactions across the BCS-BEC crossover induced by a syn- thetic Rashba spin-orbit coupling, Phys. Rev. A 86, 053617 (2012).

[28] To achieve a reliable numerical convergence, we change the limits of the radial $d q$ integration from $[0, \infty]$ to $[1,-1]$ via the change of variables $q^{\prime}=2 /(q+1)-1$, i.e., $\int_{0}^{\infty} f(x) d x=$ $2 \int_{-1}^{1} f[2 /(x+1)-1] d x /(x+1)^{2}$. 